\title{
Analyzing Overdispersed Antenatal Care Visits of Pregnant Women in Bangladesh: Negative Binomial Regression Model
}

\author{
Nasrin Sultana* and Wasimul Bari
}

Department of Statistics, Dhaka University, Dhaka-1000, Bangladesh

(Received: 3 January 2017; Accepted: 27 February 2017)

\section{Abstract}

The main aim of this paper is to find out the potential determinants of antenatal care visits of women in Bangladesh during their pregnancy. The data set was extracted from Bangladesh Demographic and Health Survey, 2014 and overdispersion is found to be present in the data set. To take
the overdispersion into account, negative binomial regression model has been used. A number of socioeconomic and demographic variables were observed to have significant impact on the antenatal care visit. Results are explained using incidence rate ratio.

Keywords: ANC visits; Dispersion parameter; Incidence rate ratio; Negative Binomial regression; Overdispersed count data.

\section{Introduction}

There has been an increasing interest in modeling the count data in many areas of application such as biology, epidemiology, public health, actuarial science, demography, automobile insurance, life insurance, biostatistics or physical applications. Analysis of the count data in the presence of a set of covariates with the Poisson regression (PR) model is common in practice. But PR model is usually found to be too restrictive because of its equidispersion assumption i.e. population mean and variance are assumed to be equal. In practice, often count data exhibit over dispersion, i.e., variance exceeds the mean of the counts. Thus, PR model for such overdispersed count data may not produce valid inference about the parameters of interest. The negative binomial (NB) regression model has been suggested as an alternative to the Poisson model when there is evidence of over-dispersion $^{1-2}$. In this paper, an attempt has been made to find out potential risk factors of antenatal care (ANC) visits for the pregnant women in Bangladesh using NB regression model.

The increasing attention to maternal health globally has concentrated on the reduction of maternal mortality, but the progress in reducing maternal mortality has been slow in developing countries like Bangladesh. Antenatal care is an important determinant of high maternal mortality rate and one of the basic components of maternal health care on which the life of mothers and babies depend. Evidence suggests that ANC identifies and manages complications during pregnancy and thus, improves pregnancy outcome ${ }^{3}$.The World Health Organization (WHO) recommends at least four ANC visits under normal circumstances of pregnancy to ensure a safe motherhood ${ }^{4}$.Therefore, it becomes an interest to find out the factors which regulate the number of ANC visits of pregnant women in such developing countries. In this paper, NB regression model has been used to identify the important factors responsible for ANC visits during the pregnancy of women in Bangladesh.

Hossain et al. ${ }^{5}$ found the contribution of socio- demographic factors on ANC visits using multiple classification analysis. Hierarchical logistic regression model was used by Khanal et al. $^{6}$ to investigate the factors associated with the underutilization of ANC of pregnant women in Timor and it was

*Author for correspondence. e-mail: nasrin_sultana@du.ac.bd found that wealth index, maternal education, paternal education and permission to visit health facility had significant effects on the under-utilization of ANC. Gupta et al. ${ }^{7}$ conducted multivariate logistic regression analysis to identify the factors associated with four or more ANC visits in Tanzania. It was found that quality of services, testing and counseling for HIV, receiving doses of Fansidar for preventing malaria during ANC, educational status, type of residence, marital status of women, distance to health facility, timing of first ANC visit and woman's desire to avoid pregnancy were associated with at least four ANC utilization. Kabir and $\mathrm{Khan}^{8}$ assessed the related factor for ANC of pregnant women in urban slums of Bangladesh. Their study revealed that education and economic condition of the respondents had strong association with the ANC received by the respondents. Shahjahan et al. ${ }^{9}$ performed logistic regression analysis to examine the association between use of ANC services and socio-demographic variables of rural women in Bangladesh and found that birth order, access to mass media and education level had significant effects on the use of ANC services. Islam and Odland $^{10}$ reported that the respondent's place of residence, age, level of education, distance to the service centers and exposure to any mass media were the significant determinants of antenatal and postnatal care visits among indigenous community. Using logistic regression model, Rahman et al. ${ }^{11}$ found that mother's education, place of treatment, husband's occupation, family's income, and exposure to mass media had highly significant effects on ANC seeking behavior among slum mothers during their las pregnancy. Simkhadaet al. ${ }^{12}$ identified the factors affecting antenatal care uptake. Their study showed that maternal education, husband's education, marital status, availability of care, cost, household income, women's employment, media exposure and having a history of obstetric complications had significant effects on receiving the ANC by pregnan women.

For the purpose of analysis, in this paper, data are extracted from Bangladesh Demographic and Health Survey (BDHS), 2014 in order to find out the potential determinants of ANC visits of pregnant women in Bangladesh. This paper consists of four sections. Data and bivariate analysis are discussed briefly in Section 2. NB regression model and findings of regression analysis are presented in Section 3. Finally, Section 4 includes the conclusion of this research work. 


\section{Data and Bivariate Analysis}

BDHS, 2014 data were collected using two-stage stratified sampling design. In the first stage, 600 enumeration areas (EAs) were selected independently with probability proportional to the EA size in each sampling stratum. The survey selected 207 EAs in urban areas and 393 EAs in rural areas. A complete household listing operation was then carried out in all the selected EAs to provide a sampling frame for the second stage selection of households. In the second stage of selection, 30 households per EA were selected with an equal probability systematic selection from the newly created household listing. The survey was conducted in 18,000 residential households, 6,210 in urban areas and 11,790 in rural areas. All ever-married women aged 15-49 who are usual members of the selected households or who spent the night before the survey in the selected households are eligible for the female survey.

The main objective of this paper is to examine the factors that are associated with the ANC visits of women during their pregnancy in Bangladesh. To reflect the recent scenario of ANC visits, information obtained only from women who had last live birth five years preceding the 2014 BDHS was considered, resulting a data set of size 6957. Based on literature, a number of demographic, socio-economic and community service related variables such as region, type of place of residence, educational attainment, wealth index, birth order, mother's age at birth, exposure of media, women empowerment and aware of community clinic have been considered as explanatory variables. Information on exposure of media and women empowerment was computed combining related covariates given in the dataset. The mothers who read newspapers or magazine or listen to radio or watch television at least once in a week were categorized as exposed to media. In the study, women were considered as empowered if they took decision alone on any one of four type decisions: their own health care, major household purchases, their child's health care, and visits to their family or relatives. Deleting all the missing values in the selected variables, finally the data set consists of 4421 observations. One-way analysis of variance (ANOVA) is performed to determine whether there are any significant differences among the mean number of ANC visits of two or more groups of an explanatory variable and the results are presented in the Table 1.

It is found that region, type of place of residence, educational attainment, wealth index, birth order, exposure of media and mother's age at birth have highly significant association (p-value $<0.001$ ) with the number of ANC visits of pregnant women. Again, women empowerment and aware of community clinic are significantly associated with ANC visits at $2 \%$ and $9 \%$ level of significances, respectively. More specifically, the highest mean number of ANC visits (3.46) is found in Khulna and the lowest one (2.03) in Sylhet division. It is observed that the mean number of ANC visits is higher in urban areas (3.68) than rural areas (2.35). Average ANC visits during pregnancy are the lowest for women having no education (1.47); with the increase in education level, increase in ANC visits are also observed.
Again, women belonging to rich family are observed to take the highest average ANC (3.81) visits and women from poo family receive the lowest average ANC visits (1.84). It is clear that the average number of ANC visits is highest (3.15) for the first birth, whereas it is 2.78 for the second to third birth and becomes the lowest (1.68) for the third birth or above.

Table 1. Average number of ANC visits by the selected covariates

\begin{tabular}{|c|c|c|}
\hline Covariate & $\begin{array}{l}\text { Mean number of } \\
\text { ANC visits } \\
(95 \% \text { CI) } \\
\end{array}$ & p-value \\
\hline Region & & $<0.001$ \\
\hline Barisal & $2.45(2.25,2.66)$ & \\
\hline Chittagong & $2.60(2.43,2.77)$ & \\
\hline Dhaka & $3.04(2.86,3.22)$ & \\
\hline Khulna & $3.46(3.22,3.69)$ & \\
\hline Rajshahi & $2.83(2.59,3.06)$ & \\
\hline Rangpur & $3.21(3.00,3.41)$ & \\
\hline Sylhet & $2.03(1.86,2.21)$ & \\
\hline $\begin{array}{l}\text { Type of place of } \\
\text { residence }\end{array}$ & & $<0.001$ \\
\hline Urban & $3.68(3.54,3.83)$ & \\
\hline Rural & $2.35(2.26,2.43)$ & \\
\hline $\begin{array}{l}\text { Educational } \\
\text { attainment }\end{array}$ & & $<0.001$ \\
\hline No education & $1.47(1.32,1.62)$ & \\
\hline Primary & $2.08(1.95,2.20)$ & \\
\hline Secondary & $3.07(2.96,3.17)$ & \\
\hline Higher & $4.72(4.47,4.97)$ & \\
\hline Wealth index & & $<0.001$ \\
\hline Poor & $1.84(1.74,1.94)$ & \\
\hline Middle & $2.49(2.34,2.65)$ & \\
\hline Rich & $3.81(3.69,3.94)$ & \\
\hline Birth order number & & $<0.001$ \\
\hline Ist birth & $3.15(3.03,3.27)$ & \\
\hline 2nd-3rd birth & $2.78(2.67,2.90)$ & \\
\hline$>3$ rd birth & $1.68(1.52,1.84)$ & \\
\hline Mother's age at birth & & 0.002 \\
\hline Age $<=20$ & $2.74(2.61,2.86)$ & \\
\hline $20<$ Age $<35$ & $2.84(2.74,2.94)$ & \\
\hline Age $>=35$ & $2.11(1.75,2.47)$ & \\
\hline Exposure of media & & $<0.001$ \\
\hline Exposed & $3.37(3.28,3.47)$ & \\
\hline Non-exposed & $1.79(1.69,1.90)$ & \\
\hline Women empowerment & & 0.011 \\
\hline Yes & $2.96(2.80,3.13)$ & \\
\hline No & $2.73(2.64,2.81)$ & \\
\hline $\begin{array}{l}\text { Aware of community } \\
\text { clinic }\end{array}$ & & 0.082 \\
\hline Yes & $2.68(2.54,2.81)$ & \\
\hline No & $2.82(2.73,2.91)$ & \\
\hline
\end{tabular}

Moreover, the highest average number of ANC visits (2.84) is observed for women aged between 20 and 35 years at 
birth. Women aged less than or equal to 20 years at birth take 2.74 average number of ANC visits and it is the lowest (2.11) for women aged greater than or equal to 35 years at birth. The results depict that mean number of ANC visits of women who are exposed to media is 3.37 , whereas it is 1.79 for women who are not exposed to media. It is also observed that the average ANC visits during pregnancy for women who are involved with taking important decisions is 2.96 and it is 2.73 for women who are not involved at all. Furthermore, the average number of ANC visits of women who are aware of community clinic is 2.68 , whereas it is 2.82 for the women being not aware of that. This evidence, however, is not statistically strong as p-value is 0.082 .

III. Negative Binomial Regression Model: Analyzing ANC Visits

To model the overdispersed count data, one may start with a Poisson regression model and add a multiplicative random effect $U$ to represent unobserved heterogeneity. This leads to the Negative Binomial regression model. Suppose that the conditional distribution of the count response, $Y$ given an unobserved variable, $U$ is Poisson with mean and variance equal to $\lambda u$. That is, $\mathrm{Y} \mid \mathrm{U} \sim \operatorname{Poisson}(\lambda \mathrm{u})$. For computational convenience, one may assume that $U$ has a gamma distribution with shape parameter, $\alpha$ and scale parameter, $\alpha^{-1}$, i.e. $U \sim \operatorname{Gamma}\left(\alpha, \frac{1}{\alpha}\right)$. Therefore, the unconditional distribution of the response $Y$ is negative binomial distribution, which is derived as follows

$$
\begin{aligned}
& f(y ; \mu, c) \\
= & \int_{0}^{\infty} \frac{e^{-\lambda u}(\lambda u)^{y}}{y !} \frac{1}{(\alpha-1) !\left(\alpha^{-1}\right)^{\alpha}} u^{\alpha-1} e^{-u /\left(\alpha^{-1}\right)} d u \\
= & \frac{\left(y+c^{-1}-1\right) !}{y !\left(c^{-1}-1\right) !}\left(\frac{1}{1+c \mu}\right)^{c^{-1}}\left(\frac{c \mu}{1+c \mu}\right)^{y}
\end{aligned}
$$

$y=0,1,2, \cdots \cdots$, where $c=\alpha^{-1}>0$ is the overdispersion parameter and $\mu=\lambda>0$. It can be shown that unconditional mean and variance have the form, $E(Y)=$ $\mu$ and $\operatorname{Var}(Y)=\mu+c \mu^{2}$, respectively. It implies that variance is larger than mean as $c>0$. Hence the NB distribution allows overdispersion in contrast to the Poisson distribution.

Regression analysis helps one understand how the typical value of the dependent variable changes when any one of the independent variables is varied keeping all other independent variables fixed. In generalized linear model (GLM), the function of mean response, $\mu=E(Y)$ is modeled in terms of a set of covariates and regression parameters. One may use the log-link function to construct the GLM for negative binomial response. Utilizing the log-link function allows a direct comparison with the Poisson model, which is NB with $c=0$ (Hilbe 2011) ${ }^{13}$.Thus, the GLM for negative binomial response can be written as $\ln \mu_{i}=x_{i}^{\prime} \beta, \quad i=$ $1 \cdots n$,where $x_{i}=\left(x_{i 1}, x_{i 2}, \ldots, x_{i p}\right)$ and $\beta=\left(\beta_{1}, \beta_{2}, \ldots, \beta_{p}\right)$ are the $p \times 1$ vector of covariates and regression coefficients, respectively. Let $\theta=\left(\beta^{\prime}, c\right)^{\prime}$ be the $(p+1) \times 1$ vector of parameters to be estimated. One may estimate $\theta$ by maximizing the log-likelihood function $l(\theta ; y)$ given as

$$
\begin{gathered}
l(\theta ; y)=\sum_{i=1}^{n}\left[y_{i} \ln \left(\frac{c \mu_{i}}{1+c \mu_{i}}\right)-\frac{1}{c} \ln \left(1+c \mu_{i}\right)\right. \\
\left.+\ln \left(\frac{\left(y+c^{-1}-1\right) !}{y !\left(c^{-1}-1\right) !}\right)\right] .
\end{gathered}
$$

For negative binomial regression model, rather than reporting regression coefficient, one may measure the effect of the independent variable on the dependent variable through the incidence rate ratio (IRR) for the convenience of interpretation. The IRR for the effect of a covariate, $x_{j}$ (either qualitative or quantitative) can be obtained as $I R R_{j}=\exp \left(\beta_{j}\right) ; j=1, \cdots, p$, if the regression model does not involve any interact term involving $x_{j}$.

Several independent variables found to have significan associations with the number of ANC visits in bivariate analysis such as region, type of place of residence, educational attainment, wealth index, birth order, mother's age at birth, exposure of media, women empowerment and aware of community clinic are included in the regression model. The descriptive statistics for the number of ANC visits are given in Table 2, which depicts that the mean number of ANC visits is 2.78 and the variance is 6.54. Thus, variance of ANC visits data exceeds its mean indicating that the data are over dispersed. For this reason, the negative binomial regression model is used to analyze ANC visits data in the presence of a set of covariates in order to determine the significant effect of covariates on the ANC response variable.

Table 2. Descriptive statistics for number of ANC visits in the study

\begin{tabular}{cc}
\hline Sample characteristics & Number of ANC visits \\
\hline Sample size & 4421 \\
Sample mean & $\mathbf{2 . 7 8}$ \\
Sample variance & $\mathbf{6 . 5 4}$ \\
\hline
\end{tabular}

The results obtained from the NB regression model are presented in Table 3 along with estimated incidence rate ratios (IRRs). It is observed that the average number of ANC visits of women from Khulna and Rangpur region is respectively $17 \%$ and $23 \%$ higher than the women in Dhaka. Again, women from Barisal, Chittagong, Rajshahi and Sylhet take respectively $7 \%, 13 \%, 5 \%$ and $18 \%$ less mean number of ANC visits compared to the women in Dhaka region, though the effects of Barisal ( $\mathrm{p}$-value $=0.126$ ) and Rajshahi (p-value $=0.322$ ) region are not statistically significant. The result also shows a highly significant influence of type of place of residence on the number of ANC visits. Women residing in urban areas receive $20 \%$ more mean ANC than women in rural areas. There is a clear significant evidence of increasing the number of ANC visits with the increasing level of educational attainment of mother. IRR for mother's education reveals that the women with higher education have $91 \%$ greater mean number of 
ANC visits compared to the women having no education. Also, the women with primary and secondary education seek $26 \%$ and $56 \%$ more mean number of ANC visits respectively compared to the women having no education. The average number of ANC visits for the women of poor family and rich family are significantly found to be $10 \%$ less and $27 \%$ more respectively compared to the women from middle class family.

Table 3. Impact of socio-economic and demographic variables on ANC visits along with p-values and IRRs, obtained from NB regression model

\begin{tabular}{|c|c|c|c|c|}
\hline Covariate & Estimate & Std. error & p-value & IRR \\
\hline Intercept & 0.356 & 0.066 & $<0.001$ & - \\
\hline \multicolumn{5}{|l|}{ Region } \\
\hline Barisal & -0.076 & 0.050 & 0.126 & 0.93 \\
\hline Chittagong & -0.145 & 0.042 & $<0.001$ & 0.87 \\
\hline Dhaka & - & - & - & \\
\hline Khulna & 0.159 & 0.046 & $<0.001$ & 1.17 \\
\hline Rajshahi & -0.047 & 0.048 & 0.322 & 0.95 \\
\hline Rangpur & 0.209 & 0.047 & $<0.001$ & 1.23 \\
\hline Sylhet & -0.203 & 0.047 & $<0.001$ & 0.82 \\
\hline \multicolumn{5}{|c|}{ Type of place of residence } \\
\hline Rural & - & - & - & - \\
\hline Urban & 0.187 & 0.030 & $<0.001$ & 1.20 \\
\hline \multicolumn{5}{|l|}{ Educational attainment } \\
\hline No education & - & - & - & - \\
\hline Primary & 0.232 & 0.051 & $<0.001$ & 1.26 \\
\hline Secondary & 0.444 & 0.051 & $<0.001$ & 1.56 \\
\hline Higher & 0.645 & 0.061 & $<0.001$ & 1.91 \\
\hline \multicolumn{5}{|l|}{ Wealth index } \\
\hline Poor & -0.107 & 0.040 & 0.008 & 0.90 \\
\hline Middle & - & - & - & \\
\hline Rich & 0.241 & 0.038 & $<0.001$ & 1.27 \\
\hline \multicolumn{5}{|l|}{ Birth order number } \\
\hline Ist birth & 0.093 & 0.035 & 0.007 & 1.10 \\
\hline 2nd-3rd birth & - & - & - & - \\
\hline$>3$ rd birth & -0.188 & 0.048 & $<0.001$ & 0.83 \\
\hline \multicolumn{5}{|l|}{ Mother's age at birth } \\
\hline Age $<=20$ & -0.120 & 0.035 & $<0.001$ & 0.89 \\
\hline $20<$ Age $<35$ & - & - & - & \\
\hline Age $>=35$ & 0.026 & 0.080 & 0.749 & 1.03 \\
\hline \multicolumn{5}{|l|}{ Exposure of media } \\
\hline Non-exposed & - & - & - & \\
\hline Exposed & 0.208 & 0.035 & $<0.001$ & 1.23 \\
\hline \multicolumn{5}{|l|}{ Women empowerment } \\
\hline No & - & - & - & - \\
\hline Yes & 0.048 & 0.031 & 0.121 & 1.05 \\
\hline \multicolumn{5}{|c|}{ Aware of community clinic } \\
\hline No & - & - & - & - \\
\hline Yes & 0.060 & 0.029 & 0.039 & 1.06 \\
\hline Dispersion parameter & 0.329 & 0.017 & $<0.001$ & \\
\hline
\end{tabular}

The effect of birth order was also found to be significantly associated with ANC visits. To be specific, the mean number of ANC visits of women is $10 \%$ higher and $17 \%$ lower during the first birth and above the third birth pregnancy, respectively compared to the second to third birth pregnancy.

-2 log-likelihood $=18020.291$ 
Again, it is found that women aged less than or equal to 20 years and greater than or equal to 35 years have $11 \%$ (pvalue $<0.001)$ less and $3 \%(\mathrm{p}$-value $=0.749)$ more mean number of ANC visits respectively than the women aged 2035 years at birth though women aged greater than or equal to 35 years at birth has no significant effect on ANC visits. The reported IRR for media exposure confirms that on the average, women who are exposed to media receive $23 \%$ higher ANC visits compared to the women being nonexposed. Furthermore, the mean number of ANC visits for women involving in taking any important decisions is observed to be $5 \%$ higher compared to the women not involving in decision making though women empowerment has no statistically significant effect on ANC visits. One can also observe that the IRR for aware of community clinic is 1.06 (p-value $=0.039)$ which implies that women being aware of community clinic have $6 \%$ more mean ANC visits during pregnancy compared to the women who were not aware at $5 \%$ level of significance.

The estimate of dispersion parameter is found to be 0.329 , far from zero, with p-value $<0.001$. It indicates that the ANC visits data are significantly over dispersed and hence the application of NB regression model to analyze this data set has become a reasonable choice and made the inference valid.

\section{Conclusion}

The results obtained from the NB regression model using BDHS, 2014data has revealed that region, type of place of residence, educational attainment of women, wealth index, birth order, mother's age at birth, exposure of media and aware of community clinic have significant effects on the number of ANC visits. Among these significant determinants of ANC visits, birth order has negative effect which indicates that number of ANC visits decreases with the increase in birth order. The positive effects of other significant determinants imply that women residing in urban areas take higher number of ANC visits compared to rural areas; the number of receiving ANC visits during pregnancy increases with the increase in educational attainment of the women; women belonging to rich family receive the highest ANC visits during their pregnancy compared to the women from poor and middle class family; the number of ANC visits is higher for women who are exposed to media and women being aware of community clinic take higher number of ANC visits than those who were not aware of that. In order to reduce the maternal and child morbidity and mortality in Bangladesh, one may suggest some recommendations to increase the ANC visits based on the results obtained in this study. Awareness should be built among the women aged less than or equal to 20 years at birth and among the women who are not aware of community clinic so that they can take more ANC services during pregnancy. Also, the women at their higher birth order pregnancy should be encouraged to receive more medical care for the safe motherhood. Moreover, basic maternal health care services should be provided to the women belonging to poor family and the women residing in rural areas. Again, the importance of ANC visits of pregnant women should be emphasized among the women living in Chittagong and Sylhet region. Female education in Bangladesh needs to be paid more attention to increase the ANC visits of pregnant women. Again, access to media of women should be facilitated for better access to ANC services during pregnancy.

\section{References}

1. Paternoster, R. and R. Brame, 1997. Multiple Routes to Delinquency? A Test of Developmental and General Theories of Crime. Criminology, 35, 45-84.

2. Osgood, D. W., 2000. Poisson-Based Regression Analysis of Aggregate Crime Rates. Journal of Quantitative Criminology, 16(1), 21-43.

3. Raatikainen K., N. Heiskanen and S. Heinonen, 2007. Underattending free antenatal care is associated with adverse pregnancy outcomes. BMC Public Health, 7, 268.

4. National Institute of Population Research and Training (NIPORT), Mitra and Associates, and ICF International. Bangladesh Demographic and Health Survey 2014 Dhaka,Bangladesh and Calverton, Maryland, USA: Mitra and Associates, and ICF International, 2016.

5. Hossain, M. K., M. R. Islam, M. N. Khan and M. R. Ali, 2015 Contribution of Socio-Demographic Factors on Antenata Care in Bangladesh: Modeling Approach. Public Health Research, 5(4), 95-102, doi: 10.5923/j.phr.20150504.03.

6. Khanal, V., J. L. N. Brites da Cruz, S. R. Mishra, R. Karkee and A.H. Lee, 2015. Under-utilization of antenatal care services in Timor-Leste: results from Demographic and Health Survey 2009-2010. Biomedical Center Pregnancy Childbirth, 15, 211.

7. Gupta, S., G. Yamada, R. Mpembeni, G. Frumence, Jennifer A. Callaghan Koru, R. Stevenson, N. Brandes and A. H.Baqui, 2014. Factors Associated with Four or More Antenatal Care Visits and Its Decline among Pregnant Women in Tanzania between 1999 and 2010. Journal of Public Library of Science, one, 9(7), e101893.

8. Kabir, R. and H. T. A. Khan, 2013. Utilization of Antenatal care among pregnant women of Urban Slums of Dhaka City, Bangladesh. Journal of Nursing and Health Science, 2(2), 15-19.

9. Shahjahan, M., H. A. Chowdhury, J. Akter, A. Afroz, M. M. Rahman and M. A. Hafez, 2012. Factors associated with use of antenatal care services in a rural area of Bangladesh. South East Asia Journal of Public Health,2(2), 61-66.

10. Islam, M.R. and J.O. Odland, 2011. Determinants of antenatal and postnatal care visits among Indigenous people in Bangladesh: a study of the Mru community. Rural and Remote Health, 11(2), 1672 (online).

11. Rahman, M., R. Islam, and M. Rahman, 2010. Antenatal Care Seeking Behaviour among Slum others: A Study of Rajshahi City Corporation, Bangladesh. Sultan Qaboos University Medical Journal, 10(1), 50-56.

12. Simkhada, B., E. R. Teijlingen, M. Porter and P. Simkhada, 2008. Factors affecting the utilization of antenatal care in developing countries. Systematic review of the literature, 61(3), 244-260.

13. Hilbe, J. M., 2011. Negative Binomial Regression, second edition, Cambridge University Press, New York. 
